\title{
Article \\ Alcohol Use and Mental Health among Older American Adults during the Early Months of the COVID-19 Pandemic
}

\author{
Marisa R. Eastman ${ }^{1}$, Jessica M. Finlay ${ }^{2} \mathbb{D}$ and Lindsay C. Kobayashi ${ }^{1, * \mathbb{D}}$ \\ 1 Center for Social Epidemiology and Population Health, Department of Epidemiology, University of Michigan \\ School of Public Health, Ann Arbor, MI 48109, USA; meastma@umich.edu \\ 2 Survey Research Center, Institute for Social Research, University of Michigan School of Public Health, \\ Ann Arbor, MI 48104, USA; jmfinlay@umich.edu \\ * Correspondence: lkob@umich.edu
}

check for updates

Citation: Eastman, M.R.; Finlay, J.M.; Kobayashi, L.C. Alcohol Use and Mental Health among Older

American Adults during the Early Months of the COVID-19 Pandemic. Int. J. Environ. Res. Public Health 2021, 18, 4222. https://doi.org/10.3390/ ijerph18084222

Academic Editors: Catherine Hennessy and Elaine Douglas

Received: 22 March 2021

Accepted: 12 April 2021

Published: 16 April 2021

Publisher's Note: MDPI stays neutral with regard to jurisdictional claims in published maps and institutional affiliations.

Copyright: (c) 2021 by the authors. Licensee MDPI, Basel, Switzerland. This article is an open access article distributed under the terms and conditions of the Creative Commons Attribution (CC BY) license (https:// creativecommons.org/licenses/by/ $4.0 /)$.

\begin{abstract}
Poor mental health associated with the COVID-19 pandemic may prompt the utilization of various coping behaviors, including alcohol use. We aimed to investigate the relationships between mental health symptomatology and self-reported changes in alcohol consumption at the onset of the pandemic. Data were from the nationwide COVID-19 Coping Study of US adults aged $\geq 55$ in April and May $2020(n=6548)$. We used population-weighted multivariable-adjusted multi-nomial logistic regression models to estimate odds ratios (ORs) for the associations between mental health (of depression, anxiety, and loneliness, each) and self-reported increased alcohol consumption (vs. no change in consumption). One in ten adults $(717 / 6548 ; 11 \%)$ reported an increase in their alcohol consumption in the past week compared to their usual pre-COVID-19 drinking. Mental health symptomatology was associated with increased drinking since the pandemic onset (depression: $\mathrm{OR}=2.66,95 \% \mathrm{CI}: 1.99-3.56$; anxiety: OR $=1.80,95 \% \mathrm{CI}: 1.34-2.42$; loneliness: $\mathrm{OR}=2.45$, 95\% CI: 1.83-3.28). Participants who screened positive for all three mental health outcomes were substantially more likely to report increased alcohol consumption since the onset of the pandemic (OR $=3.87,95 \%$ CI: 2.52-5.96, vs. no mental health outcomes). This study demonstrates potentially harmful changes in alcohol intake among middle-to-older aged adults experiencing mental health symptomatology during the early months of the COVID-19 pandemic.
\end{abstract}

Keywords: older adults; alcohol use; COVID-19; mental health

\section{Introduction}

The COVID-19 pandemic has disrupted the lives of people around the world, with the potential for substantial immediate and long-term mental health consequences [1-3]. In the United States (US), shelter-in-place and social distancing orders have challenged typical avenues of social support and engagement, and early studies have observed an increased prevalence of depression, anxiety, and loneliness at all ages in the US population [4-9]. Social isolation may be one of many factors contributing to poor mental health during the pandemic, and has previously been related to adverse mental health outcomes (including depression, anxiety, and loneliness) [1,10-19]. One study conducted among adults aged $18+$ in the early months of the pandemic found the largest proportion of increased alcohol consumption among adults aged 18-39; however, adults aged 40 and over were much more likely to self-report increased alcohol use with comorbid adverse mental health symptomatology [19]. Behavioral coping mechanisms that older adults may engage in to deal with stress and social isolation (such as alcohol consumption) and their relationships with mental health during the COVID-19 pandemic remain largely unknown. This gap in evidence limits public health policy to mitigate the population mental health burden during and beyond the pandemic. 
Alcohol consumption is a behavior commonly modified as a coping mechanism in response to life stressors, and has previously been understood according to the tension reduction hypothesis $[20,21]$. The tension reduction hypothesis suggests that alcohol consumption increases in response to cumulative and acute stress exposures in order to alleviate negative emotions $[20,22,23]$. Acute social isolation, health risks, and other life disruptions induced by the COVID-19 crisis may be such stressors, especially for middle-to-older aged adults who are at elevated risk for severe morbidity and mortality due to COVID-19 and may need to isolate more intensely than younger population groups [16,24-30]. While previous studies have produced mixed results supporting the tension reduction hypothesis, consistent evidence identifies a strong and bidirectional relationship between alcohol use and depression [22,23,31-36]. Further, previous studies suggest that exposure to stress or stressful stimuli are important motivators for increased alcohol intake [23,34,37-44]. However, it is not known how alcohol consumption may have changed among middle-to-older aged US adults during the COVID-19 pandemic.

In order to address this gap in evidence regarding pandemic-associated behavioral health outcomes, we analyzed data from a national sample of US adults aged $\geq 55$ to estimate the relationships between self-reported changes in alcohol consumption with depression, anxiety, and loneliness at the onset of the COVID-19 pandemic. We hypothesized that individuals who screened positive for depression, anxiety, and loneliness would have a higher likelihood of increased alcohol consumption at the onset of the pandemic, compared to people who did not screen positive for any of these mental health indicators.

\section{Materials and Methods}

Data for this study were collected in the baseline questionnaire of the COVID-19 Coping Study from 2 April to 31 May 2020 [45]. The COVID-19 Coping Study is a national cohort study of mental health and well-being among US adults aged $\geq 55$ during the COVID-19 pandemic, conducted by the University of Michigan. A total of 6938 US adults aged $\geq 55$ from all 50 US states, the District of Columbia, and Puerto Rico completed the baseline questionnaire. The questionnaire was administered in an online format designed for computer, tablet, and smartphone interfaces through the University of Michigan's Qualtrics survey software. The COVID-19 Coping Study was approved by the Health Sciences and Behavioral Sciences Institutional Review Board at the University of Michigan (HUM00179632), and all respondents provided online informed consent prior to starting the questionnaire. Participants eligible for this analysis were those who completed the baseline questionnaire of the COVID-19 Coping Study with non-missing data on alcohol consumption, mental health outcomes, and covariates. After all appropriate exclusions, our final analytic sample included 6635 individuals ( $96 \%$ of the total sample).

\subsection{Measures}

2.1.1. Primary Outcome: Self-Reported Change in Alcohol Consumption in the Past Week

Participants were asked to indicate past-week changes in their alcohol consumption by answering the question: "over the past week, have any of your usual daily activities or behaviors changed?" in a section that "asks about your experiences and feelings related to the ongoing COVID-19 (coronavirus) pandemic." This question asked study participants to indicate whether they were engaging in 15 specific activities or behaviors, including drinking alcohol "more than usual", "about the same as usual", "less than usual" or indicating "I don't normally do this activity". Our main outcome variable preserved these four categories to indicate self-reported changes in alcohol consumption over the past week, shortly after the onset of the COVID-19 pandemic.

\subsubsection{Primary Exposures: Depression, Anxiety, and Loneliness}

Depression, anxiety, and loneliness were assessed in the questionnaire using validated research scales. Depression was assessed using the 8-item Center for Epidemiologic Studies depression scale (CES-D), adapted from the US Health and Retirement Study. The 8-item 
CES-D asks participants to indicate the presence of eight depressive symptoms "much of the time" over the past week (yes, no), and total scores of $\geq 3 / 8$ indicated a positive screen for depression. Anxiety was assessed using the 5-item Beck Anxiety Inventory (BAI), adapted from the US Health and Retirement Study Psychosocial and Lifestyle Questionnaire [46]. The 5-item BAI asks participants to rate the frequency of anxiety symptoms in the past week using a 4-point Likert response scale ranging from $1=$ "never" to $4=$ "most of the time". Total scores of $\geq 10 / 20$ indicated a positive screen for anxiety, representing the upper 25th percentile of scores in this sample. Loneliness was assessed using the 3-item UCLA Loneliness Scale, which asks participants to report how often they felt they lacked companionship, felt left out, or isolated from others in the past week using a 3-item Likert response scale ranging from $1=$ "hardly ever" to $3=$ "often". Total scores of $\geq 6 / 9$ indicated a positive screen for loneliness, representing the upper 25th percentile of scores in this sample [47].

\subsubsection{Secondary Exposure: Mental Health Burden}

The secondary exposure variable in this study was mental health burden, which was defined as the total number of positive mental health screening indicators (depression, anxiety, and loneliness) and had a range from 0 (reference) to 3.

\subsection{Covariates}

Covariates were collected in the baseline questionnaire and included: age (years), sex (male; female), race/ethnicity (non-Hispanic White; non-Hispanic Black; Hispanic/Latinx; East Asian, Native Hawaiian, and Pacific Islander; other), education (high school diploma/ equivalent or less then high school; some college or 2-year Associate's degree; 4-year college or university degree; post-graduate or professional degree), relationship status (never married; divorced/separated; widowed; married/in a relationship), pre-pandemic employment status (employed; retired; unemployed and seeking work; unable to work due to disability or health condition; homemaker/family caregiver), pre-pandemic weekly moderate-to-vigorous intensity physical activity (none; less than $30 \mathrm{~min} ; 30 \mathrm{~min}$ to $<1 \mathrm{~h} ; 1 \mathrm{~h}$ to $<1.5 \mathrm{~h}$; $1.5 \mathrm{~h}$ to $<2 \mathrm{~h}$; $2 \mathrm{~h}$ to $<2.5 \mathrm{~h} ; 2.5 \mathrm{~h}$ or more), pre-pandemic weekly alcoholic beverages consumed (0 to $10+$ ), smoking status (never smoked; former smoker; current smoker), having a friend or family member with symptoms of or diagnosed with COVID-19 (yes; no), history of physiciandiagnosed hypertension, diabetes, heart disease, asthma, chronic obstructive pulmonary disease (COPD), cancer, or other limiting, long-standing health condition (yes; no for each), and degree of pre-pandemic social isolation (low; high). Social isolation was evaluated by five binary items according to the English Longitudinal Study of Ageing social isolation index: less than monthly contact with children, less than monthly contact with family, less than monthly contact with friends, less than monthly participation in a social organization or club, and, living alone [47]. High social isolation was defined as scoring $\geq 2 / 5$ on the index [47]. Pre-pandemic alcohol consumption was assessed by asking participants, "before the COVID19 (coronavirus) pandemic, how many drinks of alcohol did you usually have per week (e.g., glasses of wine, beer, or shots of spirits/hard liquor)?" A dropdown list of integers ranging from " 0 " to " $10+$ " was provided for participants to indicate their answer.

\subsection{Statistical Analysis}

We generated population weights using data from the 2018 American Community Survey to ensure that our study sample is representative of the general US population aged $\geq 55$ based on age, sex, race, ethnicity, education, marital status, and US census region of residence [45]. All estimates in this analysis incorporate these population weights. The characteristics of the population-weighted sample, overall and according to self-reported changes in alcohol consumption were described using univariate statistics. We specified multivariable-adjusted, population-weighted multi-nomial logistic regression models with a glogit function for categorical outcome data to estimate odds ratios (ORs) and 95\% confidence intervals (CIs) for self-reported changes in alcohol consumption in the past week, 
according to anxiety, depression, loneliness, and total mental health burden as predictor variables [48]. Four separate models were estimated, one for each mental health predictor. The reference category of the alcohol outcome variable was "no change in usual drinking" in order to evaluate how each mental health predictor was related to changes in alcohol consumption, compared to those who maintained their typical consumption patterns. All models were adjusted for the confounders described in the previous section. Analyses were conducted using SAS 9.4 (Cary, NC, USA). All $p$-values were two-tailed and statistical significance was evaluated at the 0.05 level.

\section{Results}

The mean age (standard error; SE) of the population-weighted sample was 67.7 (0.2) years, and $53.5 \%$ (95\% CI: 51.5-55.4\%) of the sample was female (Table 1). Those who reported drinking more than usual in the past week at the onset of the COVID19 pandemic also reported slightly drinking more frequently prior to the pandemic (mean: 4.7 drinks/week; SE: 0.2) than those who reported no change in their alcohol consumption (mean: 4.6 drinks/week; SE: 0.1) and those who reported drinking less than usual (mean: 3.4 drinks/week; SE: 0.2; Table 1). Approximately one in five adults (18.0\%; 95\% CI: 16.6-19.5\%) reported a change in their alcohol consumption in the past week, of which $47 \%(636 / 1353)$ identified a decrease and 53\% (717/1353) acknowledged an increase in their consumption.

Table 1. Population-weighted characteristics of the sample, COVID-19 coping study, United States, April-May 2020, $\mathrm{N}=6548$.

\begin{tabular}{|c|c|c|c|c|c|}
\hline \multirow[b]{2}{*}{ Covariates } & \multirow{2}{*}{ Overall $(\mathrm{N}=6548)$} & \multicolumn{4}{|c|}{ Change in Alcohol Consumption in the Past Week, Compared to Pre-COVID-19 Drinking } \\
\hline & & $\begin{array}{l}\text { Don't Usually Do This } \\
\text { Activity }(n=2717)\end{array}$ & $\begin{array}{l}\text { Drinking Less than } \\
\text { Usual }(n=636)\end{array}$ & $\begin{array}{c}\text { No Change in } \\
\text { Drinking }(\mathrm{n}=2478)\end{array}$ & $\begin{array}{l}\text { Drinking More than } \\
\text { Usual }(\mathrm{n}=717)\end{array}$ \\
\hline & $\%(95 \% \mathrm{CI})$ & $\%(95 \% \mathrm{CI})$ & $\%(95 \% \mathrm{CI})$ & $\%(95 \% \mathrm{CI})$ & $\%(95 \% \mathrm{CI})$ \\
\hline Age, mean (SE), years & $67.7(0.2)$ & $68.5(0.4)$ & $66.2(0.6)$ & $67.7(0.3)$ & $64.7(0.5)$ \\
\hline \multicolumn{6}{|l|}{ Sex } \\
\hline Female & $53.5(51.5,55.4)$ & $60.5(57.6,63.4)$ & $46.5(40.3,52.7)$ & $44.6(41.5,47.7)$ & $58.9(53.3,64.5)$ \\
\hline \multicolumn{6}{|l|}{ Race/Ethnicity } \\
\hline Non-Hispanic White & $73.6(71.7,75.5)$ & $73.9(71.1,76.7)$ & $62.9(55.9,69.9)$ & $75.2(72.0,78.3)$ & $78.2(72.4,84.0)$ \\
\hline Non-Hispanic Black & $10.3(8.9,11.7)$ & $10.6(8.6,12.7)$ & $15.5(10.2,20.9)$ & $9.0(6.7,11.3)$ & $7.9(3.9,12.0)$ \\
\hline Hispanic/Latinx & $9.3(7.9,10.7)$ & $7.8(6.0,9.7)$ & $15.8(9.0,22.5)$ & $9.2(7.1,11.4)$ & $10.2(5.6,14.8)$ \\
\hline $\begin{array}{l}\text { East Asian/Native } \\
\text { Hawaiian/Pacific } \\
\text { Islander }\end{array}$ & $4.4(3.5,5.4)$ & $5.3(3.7,6.9)$ & $3.4(1.4,5.5)$ & $4.1(2.6,5.7)$ & $2.0(0.5,3.6)$ \\
\hline Other & $2.3(1.9,2.8)$ & $2.4(1.7,3.1)$ & $2.4(1.0,3.8)$ & $2.4(1.6,3.3)$ & $1.6(0.3,3.0)$ \\
\hline \multicolumn{6}{|l|}{ Education } \\
\hline $\begin{array}{l}\text { High School diploma } \\
\text { or less }\end{array}$ & $44.0(41.9,46.0)$ & $50.7(47.8,53.6)$ & $41.3(34.4,48.2)$ & $38.3(34.9,41.7)$ & $32.3(25.9,38.6)$ \\
\hline $\begin{array}{l}\text { Some college or } \\
2 \text { year associate } \\
\text { degree }\end{array}$ & $27.7(26.0,29.3)$ & $26.8(24.4,29.2)$ & $28.1(22.7,33.5)$ & $28.7(25.9,31.4)$ & $28.0(23.0,33.0)$ \\
\hline $\begin{array}{l}\text { 4-year college or } \\
\text { university degree }\end{array}$ & $16.3(15.3,17.3)$ & $13.4(12.0,14.7)$ & $18.4(14.9,21.8)$ & $18.2(16.5,20.0)$ & $22.6(18.7,26.6)$ \\
\hline $\begin{array}{l}\text { Postgraduate or } \\
\text { professional degree }\end{array}$ & $12.1(11.3,12.8)$ & $9.1(8.2,10.0)$ & $12.2(9.8,14.6)$ & $14.8(13.4,16.1)$ & $17.1(14.4,19.1)$ \\
\hline \multicolumn{6}{|l|}{ Relationship status } \\
\hline Single, never married & $8.2(7.2,9.2)$ & $9.0(7.5,10.6)$ & $7.7(4.6,10.9)$ & $6.9(5.2,8.5)$ & $9.6(6.3,12.9)$ \\
\hline $\begin{array}{l}\text { Single, } \\
\text { divorced/separated }\end{array}$ & $17.9(16.4,19.5)$ & $18.8(16.6,21.0)$ & $22.7(16.5,28.8)$ & $15.6(13.1,18.1)$ & $16.9(12.0,21.8)$ \\
\hline
\end{tabular}


Table 1. Cont.

\begin{tabular}{|c|c|c|c|c|c|}
\hline \multirow{2}{*}{ Covariates } & \multirow{2}{*}{ Overall $(\mathrm{N}=6548)$} & \multicolumn{4}{|c|}{ Change in Alcohol Consumption in the Past Week, Compared to Pre-COVID-19 Drinking } \\
\hline & & $\begin{array}{l}\text { Don't Usually Do This } \\
\text { Activity }(\mathrm{n}=2717)\end{array}$ & $\begin{array}{l}\text { Drinking Less than } \\
\text { Usual }(n=636)\end{array}$ & $\begin{array}{c}\text { No Change in } \\
\text { Drinking }(\mathrm{n}=2478)\end{array}$ & $\begin{array}{l}\text { Drinking More than } \\
\text { Usual }(\mathrm{n}=717)\end{array}$ \\
\hline Single, widowed & $14.6(13.1,16.2)$ & $17.1(14.6,19.7)$ & $10.3(6.0,14.6)$ & $12.6(10.3,14.8)$ & $14.0(9.1,18.9)$ \\
\hline $\begin{array}{l}\text { Married or in a } \\
\text { relationship }\end{array}$ & $59.2(57.3,61.2)$ & $55.0(52.0,57.9)$ & $59.3(52.7,65.8)$ & $65.0(61.8,68.1)$ & $59.5(53.6,65.4)$ \\
\hline \multicolumn{6}{|l|}{$\begin{array}{l}\text { Pre-COVID } \\
\text { Employment status }\end{array}$} \\
\hline Employed & $31.4(29.7,33.0)$ & $26.2(23.9,28.5)$ & $36.1(30.4,41.8)$ & $33.8(30.9,36.6)$ & $45.4(39.8,51.0)$ \\
\hline Retired & $52.7(50.8,54.6)$ & $53.8(50.9,56.7)$ & $51.8(45.5,58.2)$ & $54.2(51.0,57.3)$ & $41.4(35.6,47.1)$ \\
\hline $\begin{array}{l}\text { Unemployed and } \\
\text { seeking work }\end{array}$ & $8.1(6.9,9.2)$ & $11.1(9.1,13.1)$ & $4.0(1.4,6.6)$ & $5.4(3.7,7.2)$ & $6.5(3.4,9.5)$ \\
\hline Unable to work & $2.8(2.0,3.6)$ & $2.6(1.4,3.8)$ & $4.4(1.8,7.0)$ & $2.8(1.2,4.3)$ & $2.3(0.4,4.1)$ \\
\hline $\begin{array}{l}\text { Homemaker/Family } \\
\text { caregiver }^{\text {a }}\end{array}$ & $5.1(4.1,6.1)$ & $6.4(4.7,8.1)$ & $3.7(1.5,5.8)$ & $3.8(2.4,5.3)$ & $4.4(1.7,7.2)$ \\
\hline \multicolumn{6}{|l|}{$\begin{array}{l}\text { Pre-COVID weekly } \\
\text { physical activity }\end{array}$} \\
\hline None & $16.0(14.4,17.6)$ & $17.2(14.9,19.5)$ & $16.9(10.2,23.6)$ & $15.3(12.7,17.8)$ & $11.3(6.7,15.9)$ \\
\hline$<30 \mathrm{~min}$ & $16.5(15.0,18.0)$ & $19.9(17.4,22.3)$ & $17.4(12.4,22.3)$ & $12.0(10.0,14.0)$ & $15.2(10.6,19.7)$ \\
\hline $30 \mathrm{~min}-<1 \mathrm{~h}$ & $14.1(12.7,15.5)$ & $14.7(12.6,16.8)$ & $19.8(14.8,24.7)$ & $12.6(10.3,14.8)$ & $10.6(7.3,13.9)$ \\
\hline $1 \mathrm{~h}-<1.5 \mathrm{~h}$ & $9.4(8.3,10.5)$ & $9.3(7.7,10.9)$ & $7.1(4.6,9.6)$ & $9.5(7.5,11.5)$ & $11.9(8.3,15.5)$ \\
\hline $1.5 \mathrm{~h}-<2 \mathrm{~h}$ & $7.9(6.9,9.0)$ & $6.4(4.9,7.9)$ & $8.0(5.1,11.0)$ & $9.8(7.8,11.7)$ & $8.7(5.7,11.6)$ \\
\hline $2 \mathrm{~h}-<2.5 \mathrm{~h}$ & $7.4(6.4,8.3)$ & $6.5(5.2,7.9)$ & $8.8(5.6,12.1)$ & $8.0(6.4,9.6)$ & $7.7(4.5,10.9)$ \\
\hline $2.5+h$ & $28.7(27.1,30.3)$ & $26.0(23.6,28.4)$ & $22.0(17.9,26.2)$ & $32.8(30.0,35.6)$ & $34.6(29.5,39.7)$ \\
\hline $\begin{array}{l}\text { Pre-COVID alcoholic } \\
\text { drinks/week, } \\
\text { mean (SE) }\end{array}$ & $2.4(0.06)$ & $0.1(0.01)$ & $3.4(0.2)$ & $4.6(0.1)$ & $4.7(0.2)$ \\
\hline \multicolumn{6}{|l|}{ Smoking Status } \\
\hline Never Smoked & $47.8(45.9,49.7)$ & $51.3(48.3,54.2)$ & $46.8(40.4,53.2)$ & $44.6(41.6,47.7)$ & $42.8(37.3,48.2)$ \\
\hline Former Smoker & $38.4(36.5,40.3)$ & $35.6(32.7,38.4)$ & $38.9(32.9,45.0)$ & $41.8(38.7,44.9)$ & $39.5(34.0,44.9)$ \\
\hline Current Smoker & $13.8(12.3,15.3)$ & $13.2(11.1,15.3)$ & $14.3(9.1,19.4)$ & $13.6(10.8,16.3)$ & $17.8(12.1,23.5)$ \\
\hline \multicolumn{6}{|l|}{$\begin{array}{l}\text { Family member or } \\
\text { friend diagnosed } \\
\text { with COVID or had } \\
\text { COVID-like } \\
\text { symptoms }\end{array}$} \\
\hline Yes & $16.1(14.8,17.3)$ & $15.6(13.6,17.5)$ & $18.7(14.1,23.2)$ & $14.7(12.7,16.6)$ & $21.6(17.2,25.9)$ \\
\hline \multicolumn{6}{|l|}{$\begin{array}{l}\text { History of chronic } \\
\text { conditions }\end{array}$} \\
\hline Hypertension & $52.0(50.1,53.9)$ & $55.0(52.1,57.9)$ & $45.9(39.4,52.4)$ & $49.4(46.3,52.6)$ & $52.6(47.0,58.3)$ \\
\hline Diabetes & $17.1(15.6,18.6)$ & $20.9(18.5,23.2)$ & $16.7(12.1,21.2)$ & $5.8(11.4,16.4)$ & $9.2(5.9,12.6)$ \\
\hline Heart Disease & $9.7(8.5,10.8)$ & $10.8(8.8,12.8)$ & $11.2(7.2,15.2)$ & $8.2(6.6,9.7)$ & $7.4(4.7,10.2)$ \\
\hline Asthma & $9.8(8.6,11.0)$ & $10.0(8.0,12.0)$ & $9.1(6.3,11.9)$ & $9.3(7.2,11.4)$ & $11.6(7.7,15.5)$ \\
\hline COPD & $9.3(7.9,10.6)$ & $10.9(8.7,13.1)$ & $7.3(4.3,10.4)$ & $6.9(4.9,8.8)$ & $12.5(7.3,17.7)$ \\
\hline Cancer & $11.4(10.3,12.5)$ & $11.7(10.0,13.3)$ & $10.9(6.8,15.1)$ & $11.6(9.9,13.4)$ & $9.4(6.4,12.4)$ \\
\hline Other & $14.8(13.5,16.1)$ & $17.5(15.4,19.5)$ & $14.6(10.0,19.2)$ & $11.5(9.6,13.5)$ & $13.0(9.4,16.6)$ \\
\hline High Social Isolation & $43.1(41.2,45.1)$ & $45.1(42.2,48.0)$ & $47.5(41.1,54.0)$ & $39.4(36.2,42.6)$ & $42.0(36.1,47.9)$ \\
\hline
\end{tabular}

Abbreviations: SE, standard error; COPD, chronic obstructive pulmonary disease. ${ }^{\text {a }}$ Participants who reported being a student $(\mathrm{n}=2)$ are included in the homemaker/family caregiver category. 
Overall, 32.0\% (95\% CI: 30.3-33.8\%) of the population-weighted sample screened positive for depression, $29.3 \%$ (95\% CI: $27.5-31.1 \%$ ) screened positive for anxiety, and $29.6 \%$ (95\% CI: 27.9-31.4\%) screened positive for high levels of loneliness. Among those who screened positive for depression, $13.3 \%$ (95\% CI: 11.2-15.3\%) reported consuming more alcohol in the past week than their usual pre-pandemic levels of consumption, and $11.4 \%$ (95\% CI: 9.4-13.4\%) indicated drinking less than usual (Table 2). Among those who screened positive for anxiety, $11.8 \%$ (95\% CI: 9.8-13.8\%) reported consuming more alcohol in the past week than their usual pre-pandemic levels of consumption, and $10.4 \%(95 \%$ CI: 8.5-12.4\%) reported drinking less than usual (Table 2). Among those who were "high" in loneliness, $12.4 \%$ (95\% CI: 10.2-14.5\%) reported consuming more alcohol in the past week than their usual pre-pandemic levels of consumption, and 11.4\% (95\% CI: 9.2-13.6\%) reported drinking less than usual (Table 2).

Table 2. Self-reported changes in alcohol consumption during the early months of the COVID-19 pandemic, according to mental health status, COVID-19 Coping Study, April-May 2020, N = 6548.

\begin{tabular}{|c|c|c|c|c|c|c|c|c|}
\hline \multirow[t]{2}{*}{ Mental Health Status } & \multicolumn{2}{|c|}{$\begin{array}{l}\text { Don't Usually Do This } \\
\text { Activity }(\mathrm{n}=2717)\end{array}$} & \multicolumn{2}{|c|}{$\begin{array}{l}\text { Drinking Less than } \\
\text { Usual }(n=636)\end{array}$} & \multicolumn{2}{|c|}{$\begin{array}{c}\text { No Change in } \\
\text { Drinking }(n=2478)\end{array}$} & \multicolumn{2}{|c|}{$\begin{array}{l}\text { Drinking More than } \\
\text { Usual }(\mathrm{n}=717)\end{array}$} \\
\hline & $\%$ & $95 \%$ CI & $\%$ & $95 \%$ CI & $\%$ & $95 \%$ CI & $\%$ & $95 \%$ CI \\
\hline \multicolumn{9}{|l|}{ Depression } \\
\hline No & 47.0 & $44.6,49.4$ & 9.1 & $7.6,10.6$ & 38.1 & $35.9,40.4$ & 5.8 & $4.9,6.7$ \\
\hline Yes & 47.9 & $44.6,51.2$ & 11.4 & $9.4,13.4$ & 27.4 & $24.4,30.4$ & 13.3 & $11.2,15.3$ \\
\hline \multicolumn{9}{|l|}{ Anxiety } \\
\hline No & 46.8 & $44.5,49.1$ & 9.6 & $8.1,11.1$ & 36.9 & $34.8,39.1$ & 6.7 & $5.7,7.6$ \\
\hline Yes & 48.5 & $44.9,52.1$ & 10.4 & $8.5,12.4$ & 29.3 & $26.0,32.6$ & 11.8 & $9.8,13.8$ \\
\hline \multicolumn{9}{|l|}{ Loneliness } \\
\hline No & 46.2 & $43.9,48.6$ & 9.2 & $7.8,10.6$ & 38.2 & $35.9,40.4$ & 6.4 & $5.5,7.3$ \\
\hline Yes & 49.8 & $46.3,53.3$ & 11.4 & $9.2,13.6$ & 26.5 & $23.5,29.4$ & 12.4 & $10.2,14.5$ \\
\hline
\end{tabular}

Note: All figures shown in table are population-weighted.

Table 3 presents results from population-weighted, multivariable-adjusted, multinomial logistic regression models predicting self-reported changes in alcohol consumption in the past week during the early COVID-19 pandemic, with "no change from usual prepandemic drinking levels" as the reference category. Individuals who screened positive for either depression, anxiety, or loneliness had greater odds of increased alcohol consumption, compared to those without any of the listed mental health indicators: OR $=2.65$ (95\% CI: 1.98-3.55, vs. no change from usual drinking) for depression, OR $=1.79$ (95\% CI: $1.33-2.41$ ) for anxiety, and OR $=2.42$ (95\% CI: 1.81-3.25) for loneliness (Table 3). Those who screened positive for each of depression and loneliness had higher odds of drinking less than usual (depression: $\mathrm{OR}=1.67 ; 95 \% \mathrm{CI}: 1.23-2.25$, vs. no change from usual drinking; loneliness: $\mathrm{OR}=1.64 ; 95 \% \mathrm{CI}: 1.21-2.24$, vs. no change in usual drinking; Table 3).

Table 4 presents the count of mental health outcomes, overall and according to selfreported changes in alcohol consumption. Individuals who screened positive for no mental health outcomes (unweighted $\mathrm{n}=3251$ ) most frequently reported no change in their usual drinking (40.2\%; 95\% CI: 37.5-42.8\%), while only $23.6 \%$ (95\% CI: $18.8-28.5 \%$ ) of individuals who screened positive for all three mental health outcomes (unweighted $n=729$ ) reported no change in their usual drinking in the past week (Table 4). Those who screened positive for two (unweighted $n=1132$ ) and three (unweighted $n=729$ ) mental health outcomes most frequently reported drinking more than usual in the past week (13.1\%; $95 \%$ CI: $10.4-15.8 \%$ for two outcomes, and 15.0\%; 95\% CI: $11.3-18.8 \%$ for three outcomes; Table 4). 
Table 3. Multi-nomial logistic regression evaluating the relationship between participants' mental health status and changes in their alcohol consumption in the past week, COVID-19 Coping Study, April-May 2020, N = 6548.

\begin{tabular}{|c|c|c|c|c|c|c|}
\hline \multirow{3}{*}{ Mental Health Indicator } & \multicolumn{6}{|c|}{ Change in Alcohol Consumption (Versus no Change from Usual Consumption) } \\
\hline & \multicolumn{2}{|c|}{ Don't Usually Do This Activity } & \multicolumn{2}{|c|}{ Drinking Less than Usual } & \multicolumn{2}{|c|}{ Drinking More than Usual } \\
\hline & OR & $95 \%$ CI & OR & $95 \%$ CI & OR & $95 \% \mathrm{CI}$ \\
\hline \multicolumn{7}{|l|}{ Depression } \\
\hline No & \multicolumn{2}{|c|}{1.00 (ref) } & \multicolumn{2}{|c|}{1.00 (ref) } & \multicolumn{2}{|c|}{1.00 (ref) } \\
\hline Yes & 1.13 & $0.85,1.51$ & 1.67 & $1.23,2.25$ & 2.65 & $1.98,3.55$ \\
\hline \multicolumn{7}{|l|}{ anxiety } \\
\hline No & \multicolumn{2}{|c|}{1.00 (ref) } & \multicolumn{2}{|c|}{1.00 (ref) } & \multicolumn{2}{|c|}{1.00 (ref) } \\
\hline Yes & 1.11 & $0.82,1.50$ & 1.27 & $0.93,1.73$ & 1.79 & $1.33,2.41$ \\
\hline \multicolumn{7}{|l|}{ Loneliness } \\
\hline No & \multicolumn{2}{|c|}{1.00 (ref) } & \multicolumn{2}{|c|}{1.00 (ref) } & \multicolumn{2}{|c|}{1.00 (ref) } \\
\hline Yes & 1.23 & $0.92,1.65$ & 1.64 & $1.21,2.24$ & 2.42 & $1.81,3.25$ \\
\hline
\end{tabular}

Note: Models adjusted for age, sex, race, relationship status, education, pre-COVID employment status, history of physician-diagnosed high blood pressure, diabetes, heart disease, asthma, chronic obstructive pulmonary disease, cancer, or any other life-limiting or chronic condition, pre-COVID-19 daily physical activity, pre-COVID-19 weekly alcohol consumption, smoking status, knowing someone diagnosed or with symptoms of COVID-19, and pre-COVID-19 social isolation. All figures shown in the table are population-weighted.

Table 4. Number of mental health outcomes participants of the COVID-19 coping study screened positive for in April and May 2020 by self-reported changes in alcohol consumption after the start of the pandemic, $\mathrm{N}=6548$.

\begin{tabular}{|c|c|c|c|c|}
\hline \multirow{2}{*}{$\begin{array}{l}\text { Number of Mental Health } \\
\text { Indicators }\end{array}$} & \multicolumn{4}{|c|}{ Population Weighted Percentage $(95 \%$ CI) of Proportion } \\
\hline & $\begin{array}{l}\text { Don't Usually Do This } \\
\text { Activity }(\mathrm{n}=2717)\end{array}$ & $\begin{array}{l}\text { Drinking Less than } \\
\text { Usual }(n=636)\end{array}$ & $\begin{array}{l}\text { No change in Usual } \\
\text { Drinking }(n=2478)\end{array}$ & $\begin{array}{l}\text { Drinking More than } \\
\text { Usual }(n=717)\end{array}$ \\
\hline 0 mental health indicators & $45.2(42.4,48.0)$ & $9.2(7.4,10.9)$ & $40.2(37.5,42.8)$ & $5.5(4.4,6.5)$ \\
\hline 1 mental health indicator & $53.1(49.2,57.1)$ & $8.7(6.4,11.0)$ & $31.6(28.0,35.2)$ & $6.6(4.9,8.3)$ \\
\hline 2 mental health indicators & $45.1(40.5,49.7)$ & $11.9(9.1,14.6)$ & $30.0(25.7,34.2)$ & $13.1(10.4,15.8)$ \\
\hline 3 mental health indicators & $49.8(44.1,55.4)$ & $11.6(8.2,15.0)$ & $23.6(18.8,28.5)$ & $15.0(11.3,18.8)$ \\
\hline
\end{tabular}

Table 5 presents results from population-weighted, multi-variable multi-nomial logistic regression models predicting self-reported changes in alcohol consumption in the past week during the early COVID-19 pandemic, with no change from usual pre-pandemic drinking levels as the reference category. Those who screened positive for two mental health outcomes were more likely to report drinking more than usual $(\mathrm{OR}=2.67 ; 95 \% \mathrm{CI}$ : $1.86-3.83$, vs. no change from usual drinking) as well as drinking less than usual (OR $=1.58$; 95\% CI: 1.09-2.31, vs. no change from usual drinking) in the past week, compared to those who screened positive for no mental health outcomes (Table 5). Similarly, those who screened positive for all three mental health outcomes had higher odds of reporting a decrease in alcohol consumption $(\mathrm{OR}=2.06$; $95 \% \mathrm{CI}: 1.28,3.30$, vs. no change from usual drinking) as well as an increase in alcohol consumption (OR = 3.83; 95\% CI: 2.48, 5.92, vs. no change from usual drinking; Table 5). 
Table 5. Multi-nomial logistic regression evaluating the association between number of mental health outcomes and changes in participants' alcohol consumption in April/May 2020, COVID-19 Coping Study, N = 6548.

\begin{tabular}{|c|c|c|c|c|c|c|}
\hline \multirow{3}{*}{$\begin{array}{c}\text { Number of Mental } \\
\text { Health Outcomes }\end{array}$} & \multicolumn{6}{|c|}{ Change in Alcohol Consumption (Versus no Change from Usual Consumption) } \\
\hline & \multicolumn{2}{|c|}{ Don't Usually Do This Activity } & \multicolumn{2}{|c|}{ Drinking Less than Usual } & \multicolumn{2}{|c|}{ Drinking More than Usual } \\
\hline & OR & $95 \% \mathrm{CI}$ & OR & $95 \% \mathrm{CI}$ & OR & $95 \% \mathrm{CI}$ \\
\hline 1 indicator & 1.33 & $0.95,1.87$ & 1.17 & $0.81,1.69$ & 1.32 & $0.91,1.94$ \\
\hline 2 indicators & 1.01 & $0.73,1.41$ & 1.58 & $1.09,2.31$ & 2.67 & $1.86,3.83$ \\
\hline 3 indicators & 1.43 & $0.88,2.30$ & 2.06 & $1.28,3.30$ & 3.83 & $2.48,5.92$ \\
\hline$p$-value for trend & \multicolumn{2}{|c|}{0.21} & \multicolumn{2}{|c|}{0.0007} & \multicolumn{2}{|c|}{$<0.0001$} \\
\hline
\end{tabular}

Note: Models adjusted for age, sex, race, relationship status, education, pre-COVID employment status, history of physician-diagnosed high blood pressure, diabetes, heart disease, asthma, chronic obstructive pulmonary disease, cancer, or any other life-limiting or chronic condition, pre-COVID-19 daily physical activity, pre-COVID-19 weekly alcohol consumption, smoking status, knowing someone diagnosed or with symptoms of COVID-19, and pre-COVID-19 social isolation. All figures shown in table are population-weighted.

\section{Discussion}

In this national study of middle-aged and older U.S. adults, we observed a high prevalence of anxiety, depression, and loneliness alongside marked self-reported changes in alcohol consumption during the early months of the COVID-19 pandemic. The prevalence of anxiety, depression, and loneliness observed in our study is higher than pre-pandemic national levels; however, it is consistent with estimates from the National Poll on Healthy Aging and a mental health survey conducted by the CDC in June $2020[4,49,50]$. The specific etiology of these increases has been attributed broadly to pandemic-associated stressors; these will be discussed further based on insights provided by our results. We observed strong associations between experiencing depression, anxiety, or loneliness and increased alcohol consumption in the past week, with a dose-response relationship between overall mental health symptom burden and drinking either more or less in the past week than usual (before the COVID-19 pandemic). This research gives insight into how middleaged and older US adults used alcohol during a period of vast uncertainty and stress, when most of the US was under shelter-in-place orders with rising COVID-19 cases and death counts [51].

\subsection{Comparison to Existing Literature}

We add to previous literature on mental health and alcohol use, finding that both increases and decreases in usual alcohol were common, and were often experienced alongside mental health symptomatology among middle-aged and older US adults during the early COVID-19 pandemic, a period of collective adversity. Evidence suggests that increases in alcohol consumption are concomitant with adverse mental health outcomes following large-scale traumatic exposures, such as a natural disaster, widespread economic assault, or acts of terrorism [37-41,43,44,52-54]. Taken together, these findings generally support the tension reduction hypothesis, indicating that alcohol consumption may be used to cope with negative emotions triggered by stress [20,22,23,42]. For example, studies conducted on the immediate and long-term aftermath of the terrorist attacks on the World Trade Center on 11 September 2011 (9/11) found that adults with high 9/11-exposure or high 9/11-PTSD were at an increased risk of subsequent binge drinking [38,39,52,55-57]. Although we were unable to assess the severity of exposure to the COVID-19 pandemic or binge drinking in our study, our results corroborate previous findings by showing high proportions of adverse mental health symptomatology and notable changes in alcohol consumption in the face of myriad stressors produced by the COVID-19 pandemic.

Furthermore, it is important to give attention to the unique context of the COVID19 pandemic and consider its impact on the interpretation of our results. Unlike previous collective traumas, the COVID-19 pandemic has induced widespread social isolation, a necessary consequence of virus mitigation orders $[1,11,16,27]$. Previous research has 
shown that social isolation can lead to poor physical, cognitive, and mental health, as well as increases in alcohol use [58-61]. The physical social isolation experienced by many during the early months of the pandemic may be a common cause and likely explanation for the relationships we observed between mental health symptomatology and increased alcohol consumption.

The relationship between mental health symptomatology and the decreased alcohol consumption that we observed may be explained in the context of social and economic changes due to the COVID-19 pandemic. Profound and sudden economic or job losses may cause affected individuals to reallocate or decrease their spending, cutting out alcohol purchases [60]. Alcohol may have become unavailable in specific contexts due to closures of stores, restaurants, and bars, and limitations on the types of social activities where alcohol is often consumed may have also affected alcohol intake [60]. Some people may be less interested in consuming alcohol outside of social engagements [60]. Physical and mental health concerns posed by the risk of COVID-19 infection may also prompt decreased alcohol consumption [60]. Previous qualitative research conducted during the COVID-19 pandemic has cited concerns over amplifying negative mental health symptomatology through alcohol use, as well as desires to prioritize healthy lifestyle choices and decrease alcohol use to support the immune system as reasons for decreased alcohol consumption [62].

Our study took a novel approach in evaluating the accumulation of multiple mental health symptom burdens in relation to alcohol use. Previous literature is sparse in this area, except to acknowledge that alcohol use is a common behavior seen among individuals with mood disorders [34-37,63-65]. Our results suggest that middle-aged and older adults with multiple mental health symptomatologies are more likely to increase their alcohol consumption during periods of collective stress associated with the COVID-19 pandemic. One explanation for this finding could be that people with multiple mental health symptomatologies have a lower psychological resilience to stressors, causing them to employ different strategies for coping, such as alcohol use. These findings may be generalizable to other collective national traumas, including potential future pandemics, which is an important area for future research attention.

\subsection{Strengths and Limitations}

A limitation of this study is its cross-sectional nature, which prevents us from determining the causal direction of association between mental health and alcohol use. We did not have information on participants' mental health prior to the pandemic. However, the prevalence of depression, anxiety, and loneliness that we observed is consistent with that found in nationally representative surveys, such as the National Poll on Healthy Aging and a mental health survey conducted by the CDC in June $2020[4,49,50]$. Furthermore, our results rely on the accuracy of self-reported data. Reluctance to honestly answer questions that may be perceived negatively is a limitation of many survey-based studies. The underreporting of alcohol use or mental health symptomatology may have led our estimates to underestimate the true magnitudes of these associations. This analysis did not include region or area of residence, such as urban versus rural location. Level of geographical isolation is frequently associated with access to services and social support [66], which may impact the relationship between mental health indicators and changes in coping behaviors, such as alcohol consumption. Finally, although we were able to obtain a large sample with representation from all 50 US states, the District of Columbia, and Puerto Rico, it is not a probability-based sample. Selection bias may be present if selection into the study was differential based on mental health and alcohol use, or according to any unmeasured factors that are uncorrelated with the sociodemographic variables used to weight our sample to the US general population. Finally, our results may not be generalizable to non-Internet users, if the associations under study differ in this population group relative to Internet users.

Our study has several strengths that bolster its contribution to knowledge of middleaged and older adults' mental health and alcohol use during the early months of the 
COVID-19 pandemic. We obtained rich social, economic, and behavioral data on a large sample size of English- and Spanish-speaking participants from all 50 US states and Puerto Rico. These data were collected during a unique period in time during the first wave of the pandemic when much of the US was under shelter-in-place orders and COVID-19 case and death counts were rapidly rising. We assessed anxiety, depression, and loneliness using research validated scales that are incorporated in other national longitudinal studies of aging (such as the US Health and Retirement Study), which increases the comparability of our findings to those of other studies. Future research may build on this study by incorporating longitudinal data to understand changes in the prevalence of mental health indicators and their impact on alcohol use among middle-aged and older U.S. adults throughout the COVID-19 pandemic and after.

\section{Conclusions}

In this large cross-sectional study of middle-aged and older US adults during the first wave of the COVID-19 pandemic, we found that changes in alcohol consumption since the start of the pandemic were associated with experiencing depression, anxiety, and loneliness. These findings draw attention to an unmet need for mental health and stress-coping resources. These results highlight the relationship between mental health, stress exposure, and alcohol use, which may serve as a foundation to identify individuals at-risk for developing problematic alcohol use and tailoring interventions to better help older adults cope with stress and isolation.

Author Contributions: Conceptualization, L.C.K., M.R.E. and J.M.F.; methodology, L.C.K.; formal analysis, M.R.E.; writing - original draft preparation, M.R.E.; writing-review and editing, L.C.K., M.R.E. and J.M.F.; visualization, M.R.E.; supervision, L.C.K. All authors have read and agreed to the published version of the manuscript.

Funding: J.M.F.'s efforts were supported by the Michigan Institute for Clinical \& Health Research Postdoctoral Translational Scholar Program (UL1 TR002240-02) and National Institute on Aging Ruth L. Kirschstein National Research Service Award Individual Postdoctoral Fellowship (F32 AG064815-01).

Institutional Review Board Statement: The study was conducted according to the guidelines of the Declaration of Helsinki, and approved by the Health Sciences and Behavioral Sciences Institutional Review Board at the University of Michigan (HUM00179632).

Informed Consent Statement: Informed consent was obtained from all subjects involved in the study.

Data Availability Statement: De-identified data are available upon reasonable request following completion of a data use agreement and paper proposal form (obtained from LCK or JFM), along with appropriate institutional research ethics board approval.

Conflicts of Interest: The authors declare no conflict of interest.

\section{References}

1. Usher, K.; Bhullar, N.; Jackson, D. Life in the pandemic: Social isolation and mental health. J. Clin. Nurs. 2020, 29, 2756-2757. [CrossRef]

2. Kumar, A.; Nayar, K.R. COVID 19 and its mental health consequences. J. Ment. Health 2020, 2, 1-2. [CrossRef]

3. Javed, B.; Sarwer, A.; Soto, E.B.; Mashwani, Z.U.R. The coronavirus (COVID-19) pandemic's impact on mental health. Int. J. Health Plann. Manag. 2020, 35, 993-996. [CrossRef]

4. Czeisler, M.É.; Lane, R.I.; Petrosky, E. Mental Health, Substance Use, and Suicidal Ideation During the COVID-19 PandemicUnited States, June 24-30 2020. MMWR Morb Mortal Wkly Rep 2020. Available online: https:/ /www.cdc.gov/mmwr/volumes / 69/wr/mm6932a1.htm?s_cid=mm6932a1_w\#suggestedcitation (accessed on 11 September 2020).

5. Kujawa, A.; Green, H.; Compas, B.E.; Dickey, L.; Pegg, S. Exposure to COVID-19 pandemic stress: Associations with depression and anxiety in emerging adults in the United States. Depress. Anxiety 2020, 37, 1280-1288. [CrossRef] [PubMed]

6. de Bruin, W.B. Age Differences in COVID-19 Risk Perceptions and Mental Health: Evidence From a National U.S. Survey Conducted in March 2020. J. Gerontol. B. Psychol. Sci. Soc. Sci. 2021, 76, e24-e29. [CrossRef] [PubMed]

7. Twenge, J.M.; Joiner, T.E. Mental distress among U.S. adults during the COVID-19 pandemic. J. Clin. Psychol. 2020, 76, $2170-2182$. [CrossRef] 
8. Taquet, M.; Luciano, S.; Geddes, J.R.; Harrison, P.J. Bidirectional associations between COVID-19 and psychiatric disorder: Retrospective cohort studies of 62,354 COVID-19 cases in the USA. Lancet Psychiatry 2021, 8, 130-140. [CrossRef]

9. Yarrington, J.S.; Lasser, J.; Garcia, D.; Vargas, J.H.; Couto, D.D.; Marafon, T.; Craske, M.G.; Nileset, A. Impact of the COVID19 Pandemic on Mental Health among 157,213 Americans. J. Affect. Disord. 2021, 286, 64-70. [CrossRef]

10. Armitage, R.; Nellums, L.B. COVID-19 and the consequences of isolating the elderly. Lancet Public Health 2020, 5, e256. [CrossRef]

11. Arpino, B.; Pasqualini, M.; Bordone, V.; Solé-Auró, A. Older People's Nonphysical Contacts and Depression During the COVID19 Lockdown. Gerontologist 2020, 61, 176-186. [CrossRef] [PubMed]

12. Brooks, S.K.; Lasserbc, J.; Garci, D.; Vargas, J.H.; Couto, D.D.; Marafon, T.; Craske, M.; Niles, A. The psychological impact of quarantine and how to reduce it: Rapid review of the evidence. Lancet 2020, 395, 912-920. [CrossRef]

13. Cacioppo, J.; Hawkley, L.; Thisted, R. Perceived Social Isolation Makes me Sad. Psychol. Aging 2010, 25, 453-463. [CrossRef]

14. Tracy, M.; Norris, F.H.; Galea, S. Differences in the determinants of posttraumatic stress disorder and depression after a mass traumatic event. Depress. Anxiety 2011, 28, 666-675. [CrossRef]

15. Zhang, H.; Tang, W. Protecting older adults during social distancing Aggregated mobility data could help fight COVID19 Disinfection threatens aquatic ecosystems. Science 2020, 368, 145-146.

16. Miller, E.A. Protecting and Improving the Lives of Older Adults in the COVID-19 Era. J. Aging Soc. Policy 2020, 32, 297-309. [CrossRef]

17. Taylor, L.; Taylor, H.O.; Nguyen, R.J.; Chatters, A.W. Social Isolation, Depression, and Psychological Distress among Older Adults. J Aging Health 2018, 30, 229-246. [CrossRef]

18. Steinman, C.; Perry, M.; Perissinotto, L. Meeting the Care Needs of Older Adults Isolated at Home During the COVID-19 Pandemic. JAMA Intern. Med. 2020, 180, 819-820. [CrossRef] [PubMed]

19. Capasso, A.; Jones, A.M.; Ali, S.H.; Foreman, J.; Tozan, Y.; DiClemente, R.J. Increased alcohol use during the COVID-19 pandemic: The effect of mental health and age in a cross-sectional sample of social media users in the U.S. Prev. Med. 2020, 145, 106422. [CrossRef]

20. Conger, J. Alcoholism: Theory, problem, and challenge. Reinforcement theory and the dynamics of alcoholism. Q. J. Stud. Alcohol. 1956, 17, 296-305. [CrossRef]

21. Peirce, R.S.; Frone, M.R.; Russell, M.; Cooper, M.L.; Mudar, P. A longitudinal model of social contact, social support, depression, and alcohol use. Heal. Psychol. 2000, 19, 28-38. [CrossRef]

22. Cooper, M.L.; Frone, M.R.; Russell, M.; Mudar, P. Drinking to Regulate Positive and Negative Emotions: A Motivational Model of Alcohol Use. J. Pers. Soc. Psychol. 1995, 69, 990-1005. [CrossRef]

23. Cooper, M.L.; Russell, M.; Skinner, J.B.; Frone, M.R.; Mudar, P. Stress and Alcohol Use: Moderating Effects of Gender, Coping, and Alcohol Expectancies. J. Abnorm. Psychol. 1992, 101, 139-152. [CrossRef]

24. Pearman, A.; Hughes, M.L.; Smith, E.L.; Neupert, S.D. Age Differences in Risk and Resilience Factors in COVID-19-Related Stress. J. Gerontol. B. Psychol. Sci. Soc. Sci. 2021, 76, e38-e44. [CrossRef] [PubMed]

25. Klaiber, P.; Wen, J.H.; DeLongis, A.; Sin, N.L. The Ups and Downs of Daily Life During COVID-19: Age Differences in Affect, Stress, and Positive Events. J. Gerontol. B. Psychol. Sci. Soc. Sci. 2021, 76, e30-e37. [CrossRef] [PubMed]

26. Banerjee, D.; D'Cruz, M.; Rao, T.S. Coronavirus disease 2019 and the elderly: Focus on psychosocial well-being, agism, and abuse prevention-An advocacy review. J. Geriatr. Ment. Health 2020, 7, 4. [CrossRef]

27. Henning-Smith, C. The Unique Impact of COVID-19 on Older Adults in Rural Areas. J. Aging Soc. Policy 2020, 32, 396-402. [CrossRef] [PubMed]

28. Holmes, E.A. Multidisciplinary research priorities for the COVID-19 pandemic: A call for action for mental health science. Lancet Psychiatry 2020, 7, 547-560. [CrossRef]

29. Starke, K.R.; Petereit-Haack, G.; Schubert, M.; Kämpf, D.; Schliebner, A.; Hegewald, J.; Seidler, A. The age-related risk of severe outcomes due to covid-19 infection: A rapid review, meta-analysis, and meta-regression. Int. J. Environ. Res. Public Health 2020, $17,1-24$.

30. Shahid, Z.; Kalayanamitra, R.; McClafferty, B.; Kepko, D.; Ramgobin, D.; Patel, R.; Shekher, C.; Aggarwal, R.V. COVID-19 and Older Adults: What We Know. J. Am. Geriatr. Soc. 2020, 68, 926-929. [CrossRef]

31. Skogen, J.C.; Knudsen, A.K.; Hysing, M.; Wold, B.; Sivertsen, B. Trajectories of alcohol use and association with symptoms of depression from early to late adolescence: The Norwegian Longitudinal Health Behaviour Study. Drug Alcohol Rev. 2016, 35, 307-316. [CrossRef]

32. Paschall, M.J.; Freisthler, B.; Lipton, R.I. Moderate alcohol use and depression in young adults: Findings from a National Longitudinal Study. Am. J. Public Health 2005, 95, 453-457. [CrossRef]

33. Boden, J.M.; Fergusson, D.M. Alcohol and depression. Addiction 2011, 106, 906-914. [CrossRef]

34. Fergusson, D.M.; Boden, J.M.; Horwood, L.J. Tests of causal links between alcohol abuse or dependence and major depression. Arch. Gen. Psychiatry 2009, 66, 260-266. [CrossRef] [PubMed]

35. Flensborg-Madsen, T. Alcohol Use Disorders and Depression-The Chicken or the Egg? Addiction 2011, 106, 915-918. [CrossRef]

36. Flensborg-Madsen, T.; Mortensen, E.L.; Knop, J.; Becker, U.; Sher, L.; Grønbæk, M. Comorbidity and temporal ordering of alcohol use disorders and other psychiatric disorders: Results from a Danish register-based study. Compr. Psychiatry 2009, 50, 307-314. [CrossRef] [PubMed] 
37. Kachadourian, L.K.; Pilver, C.E.; Potenza, M.N. Trauma, PTSD, and binge and hazardous drinking among women and men: Findings from a national study. J. Psychiatr. Res. 2014, 55, 35-43. [CrossRef]

38. Boscarino, J.A.; Adams, R.E.; Galea, S. Alcohol use in New York after the terrorist attacks: A study of the effects of psychological trauma on drinking behavior. Addict. Behav. 2006, 31, 606-621. [CrossRef] [PubMed]

39. Cerda, M.; Vlahov, D.; Tracy, M.; Galea, S. Alcohol use trajectories among adults in an urban area after a disaster: Evidence from a population-based cohort study. Addiction 2008, 103, 1296-1307. [CrossRef]

40. Nordløkken, A.; Pape, H.; Wentzel-Larsen, T.; Heir, T. Changes in alcohol consumption after a natural disaster: A study of Norwegian survivors after the 2004 Southeast Asia tsunami. BMC Public Health 2013, 13, 58. [CrossRef] [PubMed]

41. Danielson, C.K.; Sumner, J.A.; Adams, Z.W.; McCauley, J.; Carpenter, M.; Amstadter, A.; Ruggiero, K. Adolescent Substance Use Following a Deadly U.S. Tornado Outbreak: A Population-Based Study of 2000 Families. J. Clin. Child Adolesc. Psychol. 2017, 46, 732-745. [CrossRef]

42. Armeli, S.; Carney, M.A.; Tennen, H.; Affleck, G.; O'Neil, T.P. Stress and alcohol use: A daily process examination of the Stressor-Vulnerability Model. J. Pers. Soc. Psychol. 2000, 78, 979-994. [CrossRef] [PubMed]

43. Vijayasiri, G.; Richman, J.A.; Rospenda, K.M. The Great Recession, somatic symptomatology and alcohol use and abuse. Addict. Behav. 2012, 37, 1019-1024. [CrossRef] [PubMed]

44. Bosque-Prous, M.; Espelt, A.; Sordo, L.; Guitart, A.M.; Brugal, M.T.; Bravo, M.J. Job loss, unemployment and the incidence of hazardous drinking during the late 2000s recession in Europe among adults aged 50-64 years. PLoS ONE 2015, 10 , e0140017. [CrossRef]

45. Kobayashi, L.C.; Oshea, B.Q.; Kler, J.S.; Nishimura, R.; Palavicino-Maggio, C.P.; Eastman, M.R.; Vinson, Y.R.; Finlay, J.M. Cohort profile: The COVID-19 Coping Study, a longitudinal mixed-methods study of middle-aged and older adults' mental health and well-being during the COVID-19 pandemic in the USA. BMJ Open 2021, 11, e044965. [CrossRef]

46. Smith, J.; Ryan, L.; Fisher, G.G.; Sonnega, A.; Weir, D. HRS Psychosocial and Lifestyle Questionnaire 2006-16; Survey Research Center, Institute for Social Research, University of Michigan: Ann Arbor, MI, USA, 2017.

47. Steptoe, A.; Shankar, A.; Demakakos, P.; Wardle, J. Social isolation, loneliness, and all-cause mortality in older men and women. Proc. Natl. Acad. Sci. USA. 2013, 110, 5797-5801. [CrossRef]

48. Zou, G. A Modified Poisson Regression Approach to Prospective Studies with Binary Data. Am. J. Epidemiol. 2004, 159, 702-706. [CrossRef] [PubMed]

49. Malani, E.; Kullgren, P.; Solway, J. National Poll on Healthy Aging (NPHA), [United States], March, 2018; ICPSR 37305; Inter-University Consortium for Political and Social Research [Distributor]: Ann Arbor, MI, USA, 2021.

50. Clarke, T.; Boersma, P.; Schiller, J. Early Release of Selected Estimates Based on Data From the 2019 National Health Interview Survey. Natl. Cent. Health Stat. 2019, 4, 1-120.

51. Raifman, S.; Nocka, J.; Jones, K.; Bor, D.; Lipson, J.; Jay, S.; Galea, J. COVID-19 US State Policy Database; Inter-university Consortium for Political and Social Research: Ann Arbor, MI, USA, 2020.

52. Garrey, S.K.; Welch, A.E.; Jacobson, M.H.; Brackbill, R.M.; Gargano, L.M. The intentional self-medication of 9/11-related PTSD symptoms with alcohol: 15 years after the disaster. Int. J. Environ. Res. Public Health 2020, 17, 5327. [CrossRef]

53. Ma, C.; Smith, T.E. Increased alcohol use after Hurricane Ike: The roles of perceived social cohesion and social control. Soc. Sci. Med. 2017, 190, 29-37. [CrossRef]

54. Mulia, N.; Zemore, S.E.; Murphy, R.; Liu, H.; Catalano, R. Economic Loss and Alcohol Consumption and Problems During the 2008 to 2009 U.S. Recession. Alcohol. Clin. Exp. Res. 2014, 38, 1026-1034. [CrossRef]

55. North, C.S.; Adinoff, B.; Pollio, D.E.; Kinge, S.; Downs, D.L.; Pfefferbaum, B. Alcohol use disorders and drinking among survivors of the 9/11 attacks on the World Trade Center in New York City. Compr. Psychiatry 2013, 54, 962-969. [CrossRef]

56. Simons, J.S.; Gaher, R.M.; Jacobs, G.A.; Meyer, D.; Johnson-Jimenez, E. Associations Between Alcohol Use and PTSD Symptoms among American Red Cross Disaster Relief Workers Responding to the 9/11/2001 Attacks. Am. J. Drug Alcohol Abuse 2005, 31, 285-304. [CrossRef]

57. Welch, A.E.; Zeig, K.C.; Maslow, C.; Cone, J.E. Frequent binge drinking five to six years after exposure to 9/11: Findings from the World Trade Center Health Registry. Drug Alcohol Depend. 2014, 140, 1-7. [CrossRef]

58. Choi, N.G.; Dinitto, D.M. Heavy/binge drinking and depressive symptoms in older adults: Gender differences. Int. J. Geriatr. Psychiatry 2011, 26, 860-868. [CrossRef]

59. Morita, T.; Tanimoto, T.; Hori, A.; Kanazawa, Y. Alcohol use disorder due to social isolation after a nuclear disaster in Fukushima. BMJ Case Rep. 2015, 2015, 10-12. [CrossRef]

60. Forehand, C.J. Social Isolation Predicting Problematic Alcohol Use in Emerging Adults: Examining the Unique Role of Existential Isolation A. Ph.D. Thesis, The University of Vermont, Burlington, VT, USA, 2018.

61. Killgore, W.D.S.; Cloonan, S.A.; Taylor, E.C.; Lucas, D.A.; Dailey, N.S. Alcohol dependence during COVID-19 lockdowns. Psychiatry Res. 2021, 296, 113676. [CrossRef]

62. Grossman, E.R.; Benjamin-Neelon, S.E.; Sonnenschein, S. Alcohol consumption during the covid-19 pandemic: A cross-sectional survey of us adults. Int. J. Environ. Res. Public Health 2020, 17, 9189. [CrossRef] [PubMed]

63. Pirkola, S.P.; Isometsa, E.; Suvisaari, J.; Aro, H.; Joukamaa, M.; Poikolainen, K.; Koskinen, S.; Aromaa, A.; Lönnqvist, J. DSM-IV mood-, anxiety- and alcohol use disorders and their comorbidity in the Finnish general population. Results from the Health 2000 Study. Soc. Psychiatry Psychiatr. Epidemiol. 2005, 40, 1-10. [CrossRef] 
64. Li, T.K.; Hewitt, B.G.; Grant, B.F. Alcohol use disorders and mood disorders: A National Institute on Alcohol Abuse and Alcoholism perspective. Biol. Psychiatry 2004, 56, 718-720. [CrossRef]

65. Prior, K.; Mills, K.; Ross, J.; Teesson, M. Substance use disorders comorbid with mood and anxiety disorders in the Australian general population. Drug Alcohol Rev. 2017, 36, 317-324. [CrossRef]

66. Finlay, J.M.; Kobayashi, L.C. Social isolation and loneliness in later life: A parallel convergent mixed-methods case study of older adults and their residential contexts in the Minneapolis metropolitan area, USA. Soc. Sci. Med. 2018, 208, 25-33. [CrossRef] [PubMed] 